\title{
An Energy-Balanced Routing Algorithm for ZigBee Audio Guide System in Ad Hoc Social Network
}

\author{
Jian Zou ${ }^{a}$, Demin $\mathrm{Li}^{\mathrm{b}}$, Min Zhang ${ }^{\mathrm{c}}$ \\ College of Information Science and Technology, Donghua University, Shanghai 201620, China \\ adaviszou@qq.com, bdeminli@dhu.edu.cn, ‘174350291@qq.com
}

Keywords: ZigBee, Audio communication, Energy-Balanced

\begin{abstract}
In order to avoid the network partitioning and nodes died too early in ZigBee audio guide system, we designed an energy-balanced routing algorithm in this paper, which can manage the rest energy of all network nodes effectively. The simulation results indicate that this algorithm can balance the entire network energy, extend the survival time of the whole network and increase the stability of the system relative to the original routing protocol.
\end{abstract}

\section{Introduction}

ZigBee wireless communication protocol is designed for those fields which have low demand for data rate. In the audio guide system, the communication burden is audio data stream. Even though the data stream is compressed by audio compression coding and also heavily, it will cause too much energy consumption. Although ZigBee wireless communication protocol has already designed sleep mechanism to save energy, the sleep mechanism only used in RFD (Reduced Function Device), all of nodes in audio guide system must be FFD (Full Function Device). And the network structure is tree which causes energy conservation unbalanced and reduces the lifetime of system rapidly. So the problem of energy is a challenge in the audio guide system which power supply by battery.

About the energy-efficient of tree network structure has discussed in related works. The paper [1] had proposed to get the newest energy distribution about its neighbors and get the mean area energy as threshold to select the routing. The paper [2] had set every router a specific delay which directly proportional to reciprocal of its rest energy, every router transfer packet after this specific time delay and the network select routing depend on which packet arrive first . The paper [6] had given an idea divide all the nodes into three different energy levels which will influence the routing process, the node relay packet or not according to their own energy level in the process of data transfer. The paper [7] has proposed DFG-TR (Destination Family Group-Tree Routing) algorithm that searches the destination node and its family from the neighbor node. If the destination node exists in the neighbor node, it immediately sends data to the destination. If not, it creates the destination node's family, if a created family exits in the neighbor node, then this is chosen as the next hop node, if not, DFG-TR finds the destination node through the pure tree routing. All of those algorithms have an advantage over traditional AODVjr [8], but the nodes closed to root node are difference to general nodes, for these nodes, those papers have no considered too much, and for nodes have many child nodes have no considered too, for those nodes have special sociality and reflect deferent social relationships and deferent social hierarchy in the network. These nodes play very important roles, and the audio guide system will appear network partitioning if you ignore the energy problem of these nodes in Ad Hoc social network.

In order to solve these problems, we proposed an energy-balanced routing algorithm in this paper. This algorithm brings in a concept named energy-conservation priority (EP) in the process of calculate energy threshold, to compare every node's rest energy with their energy threshold, so that we can select an optimal routing which can balance the energy of entire audio guide system. In this paper, we designed neighbor-list in every FFD node firstly, which used for providing neighbor node's information at the routing time. And then we defined EP and $\mathrm{E}_{\text {threshold, }} \mathrm{EP}$ is primarily use for protecting these nodes closed to root node and owned too many child nodes. $E_{\text {threshold }}$ as a threshold value used for comparing with node's rest energy so as to select an optimal routing which can get a 
result of energy-balanced. Because the $E_{\text {threshold }}$ value can't a constant, in the following section, we defined a function $\varphi(\mathrm{M})$ to update the $\mathrm{E}_{\text {threshold }}$ dynamically. Moreover, considering some nodes act as leaf nodes of tree structure needn't routing, we set them sleep mechanism to save energy consumption furthermore.

\section{Energy-Balanced routing algorithm}

Now, a lot of research about the energy problem of wireless network had been discussed and proposed much algorithm. But no matter what kind of routing algorithm cannot guarantee in any network scene to achieve optimal, different network scene, different performance be reflected. The network structure of audio guide system is tree structure with all FFD nodes, show in Fig. 1. All mentioned algorithm in this scene is not the most appropriate.

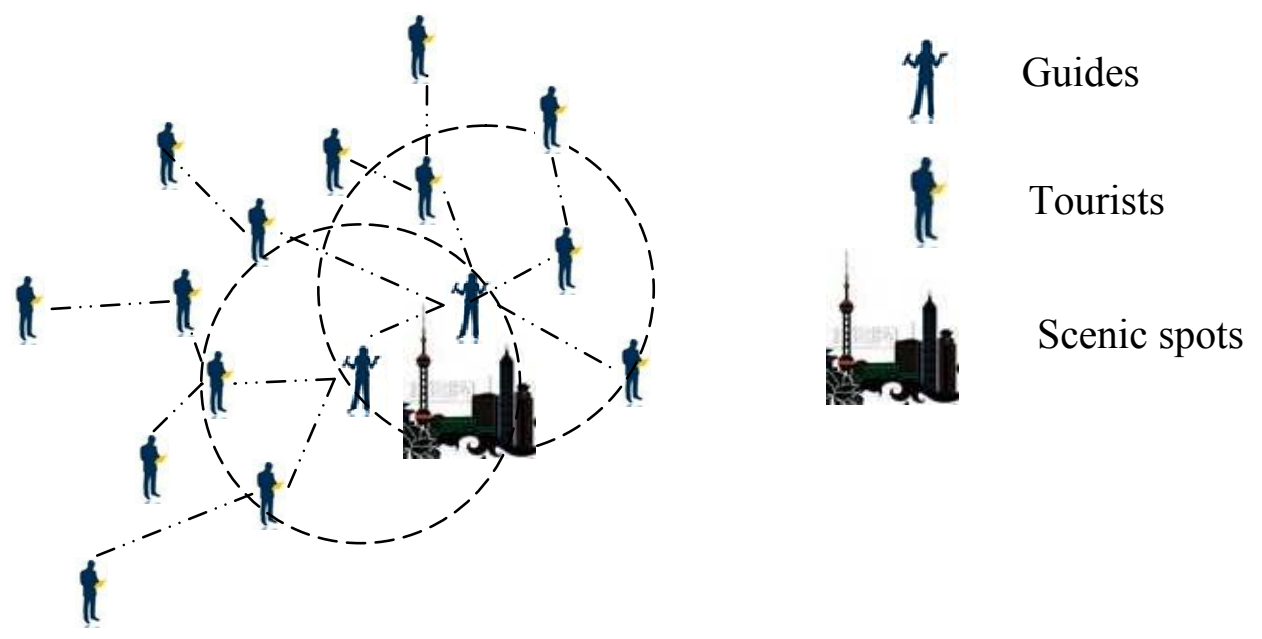

Fig. 1 Network topology of audio guide system

Neighbor-list Design. All FFD nodes can record the node information of other neighbor nodes in the network of audio guide system. Show in Table 1.

Table 1 Neighbor-list

\begin{tabular}{|l|l|l|}
\hline Neighbor ADDR & Relationship & Rest Energy(RE) \\
\hline
\end{tabular}

Neighbor ADDR indicates the network address of neighbor nodes, Relationship indicates the relationship with neighbor nodes, if Relationship $=1$, show it's a father node, Relationship $=0$, show it's a child node, and Rest Energy indicates the rest energy of neighbor nodes.

Definition of EP and $\mathbf{E}_{\text {threshold }}$. Suppose $\mathrm{N}_{0}$ is the guide transmitter, namely multicast source. $\mathrm{N}_{1}, \mathrm{~N}_{2}, \cdots, \mathrm{N}_{\mathrm{m}}$ is tourists receiver, namely multicast receiver. The audio data from $\mathrm{N}_{0}$ to $\mathrm{Nm}$ have possible paths $R_{1}, R_{2}, \cdots, R_{k}$ with $k . N_{1}, N_{2}, \cdots, N_{m-1}$ are intermediate nodes of one path, $E_{i, j}$ indicates the energy consumption that transmits packet form node $N_{i}$ to node $N_{j}$. So the energy consumption that transmits packet form node $\mathrm{N}_{0}$ to node $\mathrm{N}_{\mathrm{m}}$ can be showed by Eq.1.

$$
C_{R_{k}}=\sum E_{i, j .} \quad i, j \in\left\{\text { all nodes in } R_{k}\right\}
$$

Each path mapping a total energy consumption in mentioned possible paths $\mathrm{R}_{1}, \mathrm{R}_{2}, \cdots, \mathrm{R}_{\mathrm{k}}$ with $\mathrm{k}$, if we select the path $\mathrm{C}=\min \left\{\mathrm{C}_{\mathrm{R} 1}, \mathrm{C}_{\mathrm{R} 2}, \cdots, \mathrm{C}_{\mathrm{Rk}}\right\}$ as an optimal path which can guarantee the minimum energy consumption of the whole network, but it cannot guarantee the balance of energy consumption. For audio transmit, according to NGB (Next Generation Broadcasting Network), the maximum delay of audio communication must less than $150 \mathrm{~ms}$, and the audio data packet transmitted by ZigBee router nodes need $30 \mathrm{~ms}$ at least. So the routing hops must less than 5 hops when routing select.

Because in tree structure network, the more router nodes close to coordinator, the larger possibility that being selected as router, so as to the heavier energy consumption. And for nodes owned too many child nodes, would transmit audio data many times bring about too much energy 
consumption. But all this nodes are playing a very important role in network different to other general nodes, so we bring in a concept named energy- conservation priority (EP) here, all of above mentioned nodes that have important role in network and we give them the high priority level, and rule the higher priority nodes have, the higher energy nodes need. We define EP of node i like Eq.2.

$$
E P(i)=f(C(i), d(i))
$$

Where $\mathrm{f}$ is a function of C(i) and d(i), d(i) is the depth of node i, C(i) is the number of child nodes of node i.

Suppose every father node at most can be connected with $\mathrm{C}_{\mathrm{m}}$ child nodes, node in these child nodes at most can have $\mathrm{R}_{\mathrm{m}}$ routing nodes, maximum depth of network is $\mathrm{L}_{\mathrm{m}}$. $\mathrm{C}_{\text {skip }}$ is offset between addresses of child nodes which are allocated by their farther node whose network depth is $\mathrm{d}, \mathrm{C}_{\text {skip }}$ can be calculated by formula [3] like Eq.3.

$$
C_{\text {skip }}= \begin{cases}1+C_{m}\left(L_{m}-d-1\right) & R_{m}=1 . \\ \frac{1+C_{m}-R_{m}-C_{m} R_{m}^{L_{m}-d-1}}{1-R_{m}} & R_{m}>1 .\end{cases}
$$

In this text, we get $\mathrm{R}_{\mathrm{m}}>1$ and $\mathrm{C}_{\text {skip }}$ can be calculated by Eq. 4 .

$$
C_{\text {skip }}=\frac{1+C_{m}-R_{m}-C_{m} R_{m}^{L_{m}-d-1}}{1-R_{m}}=\frac{R_{m}^{L_{m}-1}}{R_{m}-1} C_{m} R_{m}^{-d}-\frac{C_{m}}{R_{m}-1}+1=Q C_{m} R_{m}^{-d}-P C_{m}+1 .
$$

Where $Q=\frac{R_{m}^{L_{m}-1}}{R_{m}-1}, P=\frac{C_{m}}{R_{m}-1}$.

In a ZigBee network, $\mathrm{Q}$ and $\mathrm{P}$ are constant, and $\mathrm{Q}>0, \mathrm{P}>0$. Now, $\mathrm{C}_{\text {skip }}$ is a function of node depth and maximum child nodes. This function is similar to EP we defined above very much, the smaller the depth, the greater the importance, the more the child nodes, the greater the importance. So we can define the EP (i) of node i like Eq.5.

$$
E P(i)=C(i) R_{m}^{-d(i)}-\mu C(i)+\xi .
$$

Where $\mu=\frac{P}{Q}>0, \xi=\frac{1}{Q}>0$.

We set a dynamic energy threshold $E_{\text {threshold }}$ which used to compare with node's rest energy in the network, and select an optimum route combining with the energy-balanced priority of each node. If the initial energy of each node is $\mathrm{E}_{0}$, the $\mathrm{E}_{\text {threshold }}$ can be defined like Eq.6.

$$
E_{\text {threshold }}(i)=\frac{\eta}{\varphi(M)} \times \frac{E P(i)}{E P_{\max }(i)} \times E_{0} .
$$

In Eq. $6, \eta$ is a regulatory factor which used for control the variation of speed. $\varphi(\mathrm{M})$ is a function used for dynamic update of $\mathrm{E}_{\text {threshold }}, \mathrm{M} \in[0, \mathrm{R}]$ will be detailed introduce in the following text, indicate the counts of dynamic update. $\mathrm{R}=\mathrm{E}_{0} / \mathrm{E}_{\mathrm{c}}, \mathrm{E}_{\mathrm{c}}$ is the energy consumption of being transferred by ZigBee router nodes one time.

Formation of $\varphi(\mathbf{M})$. Define two variables $\mathrm{M}_{1}, \mathrm{M}$ in the node of the Z-Stack coordinator. If the rest energy $(\mathrm{RE})$ of the node $\mathrm{i}$ in the network is less than the energy threshold $\mathrm{E}_{\text {threshold, }}$ the node $\mathrm{i}$ will send a warning signal to the coordinator, $\mathrm{M}_{1}$ add 1 after the coordinator received a warning signal. Make $\mathrm{P}=\mathrm{M}_{1} / \mathrm{N}, \mathrm{P}$ means the percentage of network nodes which energy is below the threshold value. $\mathrm{M}$ add 1 if $\mathrm{P}>\mathrm{T}(0<\mathrm{T}<1$, arbitrary set of values $)$. The added value of the $\mathrm{M}$ included in the routing request packet, whenever $M$ changes, the energy threshold of each node in the system will be updated once. After the update of the energy threshold, $\mathrm{M}_{1}$ is cleared and recounted. 
The process of $E_{\text {threshold }}$ dynamic update is $E_{\text {threshold }}$ become smaller and smaller and the decreasing degree of $\mathrm{E}_{\text {threshold }}$ is becoming smaller and smaller. So $\varphi(\mathrm{M})$ is an increasing function with $M$ at $[0, R], \varphi(M)$ grows bigger with $M$, when $M$ approaches to $R, \varphi(M)$ is infinitely great, and the increasing degree of $\varphi(\mathrm{M})$ is becoming bigger and bigger. Because $\mathrm{E}_{\text {threshold }}(\mathrm{i})$ has an inverse ratio relationship with $\varphi(\mathrm{M})$, so $\mathrm{E}_{\text {threshold }}(\mathrm{i})$ grows smaller with $\mathrm{M}$, and the decreasing degree of $E_{\text {threshold }}(i)$ is becoming smaller and smaller, and $E_{\text {threshold }}(i)$ has a direct ratio relationship with EP(i).So different network nodes at the same time, the higher priority the bigger energy threshold. It is beneficial to protect nodes that with smaller depth and more child nodes so as to avoid network partitioning efficiently. So $\varphi(\mathrm{M})$ can be defined like Eq.7.

$$
\varphi(M)=\frac{E_{0} e^{\alpha M}}{E_{0}-M E_{c}} .
$$

In Eq.7, $\alpha$ is a regulatory factor which used for control the counts of $\mathrm{M}$, so as to regulate the time slot of $M$ changes. The bigger $M$ counts, the longer time slot of $M$ changes. For example, if $\alpha=0.1$, $\mathrm{M}$ can count to 50 , else if $\alpha=0.01, \mathrm{M}$ can count to 500 , and the time slot of $\mathrm{M}$ changes is different. $\mathrm{M} \in[0, \mathrm{R}]$, we can calculate $\varphi^{\prime}(\mathrm{M})$ and $\varphi$ '’(M) like Eq.8 and Eq.9.

$$
\begin{gathered}
\varphi^{\prime}(M)=\left[\frac{\alpha}{E_{0}-M E_{c}}+\frac{E_{c}}{\left(E_{0}-M E_{c}\right)^{2}}\right] E_{0} e^{\alpha M} . \\
\varphi^{\prime \prime}(M)=\left[\frac{\alpha^{2}}{E_{0}-M E_{c}}+\frac{2 \alpha E_{c}}{\left(E_{0}-M E_{c}\right)^{2}}+\frac{2 E_{c}^{2}}{\left(E_{0}-M E_{c}\right)^{3}}\right] E_{0} e^{\alpha M} .
\end{gathered}
$$

Eq.8 and Eq.9 showed that both $\varphi^{\prime}(\mathrm{M})$ and $\varphi^{\prime \prime}(\mathrm{M})$ greater than zero, so $\varphi(\mathrm{M})$ is an increasing function with $M$ at $[0, R]$, and the increasing degree of $\varphi(M)$ is becoming bigger and bigger.

Sleep Mechanism. In the ZigBee protocol specification, FFD does not have the sleep feature. That's because FFD has the function of routing, if added the sleep mechanism, it will affect the routing discovery process of the whole network. However, in the ZigBee audio guide system which the network structure is a tree structure, there must be some of FFD nodes are not used as routers, these nodes act as a leaf node of the tree structured network. They can be added the sleep mechanism to save energy. We define a routing state flag RFlag on each FFD device. In the network operation process, if the node acts as a routing role in the network, we set RFlag=1, if the node does not act as a routing function while as a leaf node of the tree network, then we set $\mathrm{RFlag}=0$.To enable the sleep mechanism by judging the routing state RFlag of the node. If RFlag $=0$, start up the sleep mechanism, if RFlag $=1$, use the above algorithm to save energy and abandon the sleep mechanism.

\section{Simulation Results}

Energy-balanced routing algorithm is designed primarily to make the energy of the audio guied system to get a balanced effect. Considering the nodes closed to coordinator node are more likely to be selected as the routing nodes, and this nodes in the network play an important role, so we should consider the energy situation of this nodes in the simulation experiments mainly. We assume that there are 2 guide transmitters and 20 tourist receivers which have a routing function in the network, set $\mathrm{R}_{\mathrm{m}}=4, \mathrm{~L}_{\mathrm{m}}=5, \eta=1, \alpha=0.01, \mathrm{~T}=0.5, \mathrm{E}_{0}=100 \mathrm{~J}, \mathrm{E}_{\mathrm{c}}=0.01 \mathrm{~J}$, the node transmission distance set $20 \mathrm{~m}$.

The algorithm mentioned in the case of nodes have permitted residual energy at routing, if there are $\mathrm{k}$ paths to reach destination, choose the route with minimum energy overhead as the best path, it can effectively save the energy cost of the entire network. And design sleep mechanism to nodes which has no routing function, so it can save the total energy consumption of network. The total energy consumption for network simulation result is shown in Fig. 2. And the energy consumption of node simulation result is shown in Fig. 3. In this text, because we designed this algorithm primarily to protect energy consumption of these nodes which closed to root and owned too many child nodes, so in this simulation, we select the child nodes of root node. 


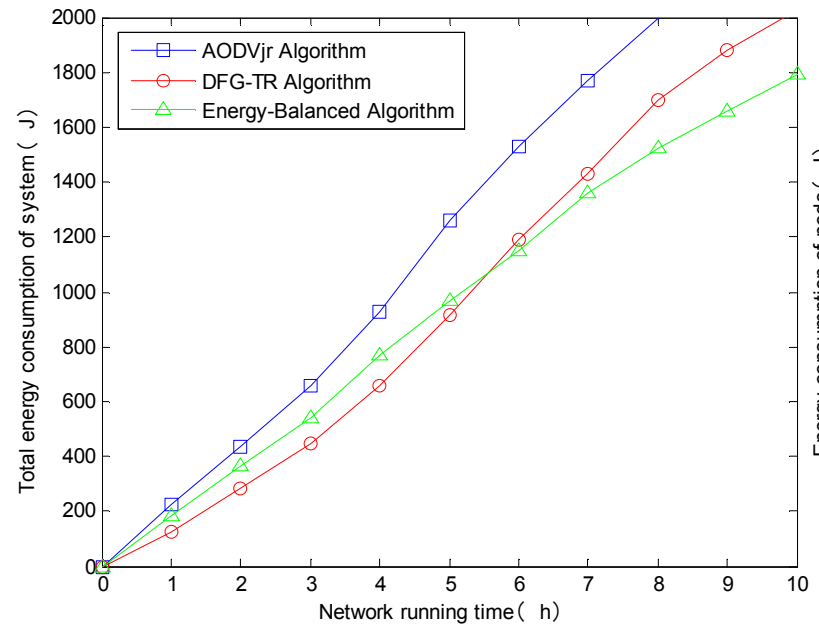

(a)

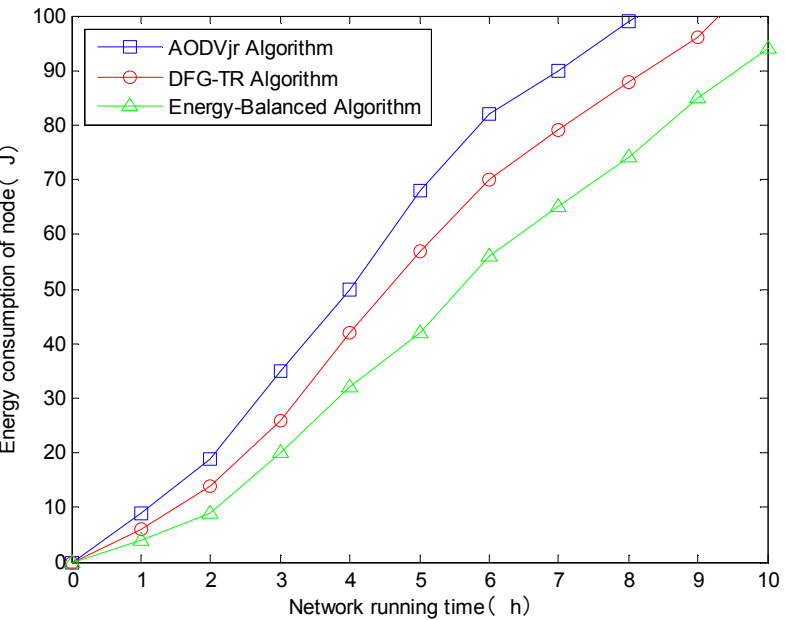

(b)

Fig. 2 (a) Total energy consumption varies with time

Fig. 3 (b) Energy consumption of node varies with time

It can be seen in the simulation results, as the network operation time increases, the network's total energy consumption also increases. The proposed algorithm combined with the minimum cost algorithm, energy-balanced mechanism and sleep mechanism algorithm, effectively reduces the total energy consumption of the entire network compared with the DFG-TR algorithm [7] and traditional AODVjr algorithm [8].

The proposed algorithm can also prolong the survival time of each node, especially give nodes high priority level which closed to root node to avoid died too early, and focus on the protection of those nodes which playing an important role in the network of routing. The survival time for node simulation results are shown in Fig. 4.

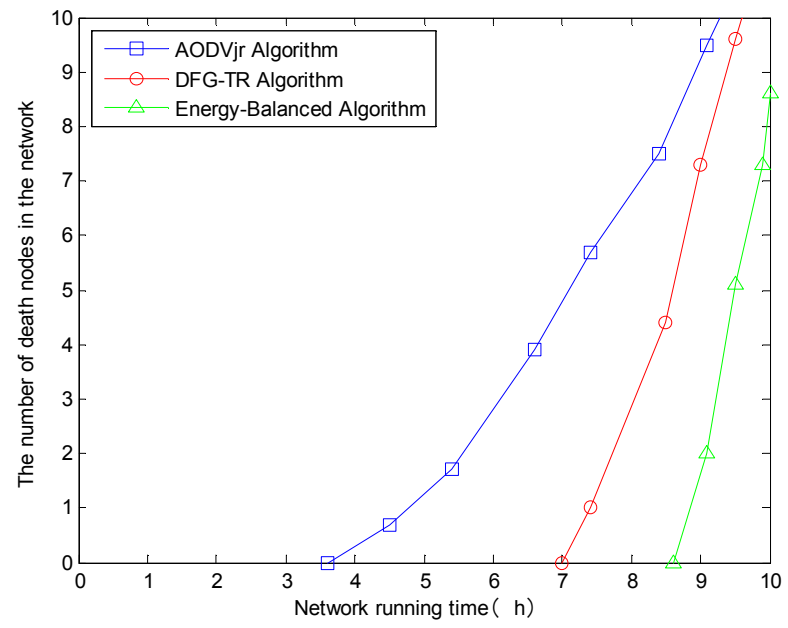

Fig. 4 The number of death nodes varies with time

It can be seen in the simulation results, as the network time increases, the number of the death nodes in the network also will increase. We can compare the start time of death node through the simulation curve, it shows traditional AODVjr algorithm [8] has a death node after 4 hours, the DFG-TR algorithm [7] has a death node after 7 hours, and then the number of the death nodes also increase substantially. With the proposed routing algorithm, there is a death node after 8.5 hours. This suggests that the proposed energy-balanced routing algorithm further prolong the survival time of the node. 


\section{Conclusions and Future work}

This paper introduces an energy-balanced routing algorithm application to the audio guide system, due to transferred data is relatively large, and the system is tree network and all nodes are FFD. Based on the characteristics of the system, we design a kind of energy-balanced algorithm. The algorithm will be priority to those who take a major role in the network routing node, and add the sleep mechanism selectively. Thus the system can control the energy costs of the whole network and the survival time of the single node effectively. Comparing this algorithm to traditional energy saving algorithm through the software simulation, it reflects the superiority of the energy-balanced algorithm from the entire energy consumption, energy consumption of one child node of root node and the survival time of a single node. All these discussions are theoretic, and in the future, we will apply this algorithm to practice to confirm the superiority of this algorithm.

\section{Acknowledgements}

This work is partially supported by NSFC granted number 71171045; National ITER Plan grant number 2010GB108004; Shanghai Key Scientific Research Project under grant number 05dz05036.

\section{References}

[1] Ran Peng, Sun Mao-heng, Zou You-min, ZigBee Routing Selection Strategy Based on Data Services and Energy-balanced ZigBee Routing. Washington DC: IEEE Computer Society(2006) 400-404.

[2] Wang Fang, Chai Qiao-lin, Ban Yan-li, Improved routing algorithm for ZigBee mesh networks. Computer Application, Vol.28(2008) 2788-2790.

[3] ZigBee Alliance: ZigBee Specification 2007(http://www.zigbee.org/Specifications.aspx) (2008) 398-399.

[4] Varun Goyal, Vatsal Mittal, Manan Aggarwal, Technique For Making ZigBee Power Efficient. International Conference on Advances in Computing, Communication and Control(2009) 92-95.

[5] Ban Yan-li, Chai Qiao-lin, Research of Routing Algorithms Based on Energy Efficient for ZigBee Network. Computer Application, Vol.28 (2009) 2792-2794.

[6] YiGong Peng, YingLi Li, ZhongCheng Lu, JinShou Yu, Method for Saving Energy in ZigBee Network. The 5th International Conference on Wireless communications, networking and mobile computing, (2009)

[7] Saeyoung Ahn, Doohyun Ko, Bumjin Kim, Sangbin Lee, Sunshin An: Energy-efficient Tree Routing Algorithm-based Destination Family Group in ZigBee Networks. The 4th International Conference on Sensor technologies and Applications, (2010)

[8] Xie Chuan, Research of AODVjr Algorithm Based on ZigBee. Computer Engineering, Vol.37 (2011)87-89. 